\title{
ON THE EXPANSION OF ANALYTIC FUNC.TIONS IN SERIES OF POLYNOMIALS AND IN SERIES OF OTHER ANALYTIC FUNCTIONS*
}

\author{
BY
}

J. L. WALSH

1. Introduction. The present paper is substantially a continuation of a previous paper $\dagger$ in which polynomial developments of an arbitrary analytic function were considered, culminating in three theorems. The first of these theorems is in essence a modification and completion of a result due to Birkhoff, a generalization of Taylor's development about the origin in the plane of the complex variable $x: \ddagger$

Theorem I. Let the functions

$$
p_{0}(x), p_{1}(x), p_{2}(x), \cdots
$$

be analytic for $|x| \leqq 1+\epsilon$, and such that on and within the circle $\gamma^{\prime}:|x|=1+\epsilon$, we have

$$
\left|p_{k}(x)-x^{k}\right| \leqq \epsilon_{k} \quad(k=0,1,2, \cdots),
$$

where the series $\sum \epsilon_{k}^{2}$ converges to a sum less than unity, and where the series $\sum \epsilon_{k}$ converges. Then there exists a set of functions $P_{k}(x)$ continuous for $|x| \geqq 1$, analytic for $|x|>1, \S$ zero at infinity, and such that

$$
\int_{\gamma} P_{k}(x) p_{i}(x) d x=\delta_{k i}=\left\{\begin{array}{l}
0, i \neq k, \\
1, i=k,
\end{array} \quad \gamma:|x|=1 .\right.
$$

If $F(x)$ is any function integrable and with an integrable square (in the sense of Lebesgue), then the two series

$$
\begin{array}{rlrl}
\sum_{k=0}^{\infty} a_{k} x^{k}, & a_{k} & =\frac{1}{2 \pi i} \int_{\gamma} F(x) x^{-k-1} d x, \\
\sum_{k=0}^{\infty} c_{k} p_{k}(x), & c_{k}=\int_{\gamma} F(x) P_{k}(x) d x,
\end{array}
$$

have on $\gamma$ (and hencell in the closed region $|x| \leqq 1$ ) essentially the same con-

* Presented to the Society, September 9, 1927; received by the editors in May, 1927.

$\dagger$ These Transactions, vol. 26 (1924), pp. 155-170. We shall refer to this paper as I.

$\$ 1$, p. 159.

\& See below, \& 2 .

|| A convergent series of constant terms dominates the term-by-term difference of series (3) and (4) for $|x|=1$ and hence for $|x| \leqq 1$. 
vergence properties, in the sense that their term-by-term difference approaches uniformly and absolutely the sum zero. In particular if $F(x)$ is continuous for $|x| \leqq 1$, analytic for $|x|<1$, and satisfies a Lipschitz condition on $\gamma$, then the series (4) converges uniformly to the sum $F(x)$ in the closed region $|x| \leqq 1$.

In I this theorem was applied, after conformal transformation, to obtain the two other theorems mentioned, the first on the expansion of an analytic function in terms of polynomials, the second including the analogue of the Laurent series. In the present paper we treat (Part A) more in detail the analogy between the two series (3) and (4), considering arbitrary series of type (4), the analogue of Abel's theorem and its converse, convergence properties on circles other than $\gamma$, and the uniqueness of expansions. In Part B we apply these results to the case of polynomials belonging to a given region, and collect the main results of the paper in Theorem IX. We consider in particular the expansion of a discontinuous function, in Theorem XI. It is found that under certain conditions Gibbs's phenomenon occurs, precisely as for Fourier's series. In Part $\mathrm{C}$ we study the use of polynomial expansions in connection with multiply-connected regions, obtaining certain results on the boundary values of analytic functions.

\section{A. Series of analytic functions}

2. Modification of proof of Theorem I. The proof of Theorem I given in I is needlessly complicated. It is perhaps worth while to present in some detail a modification, for we shall need later certain inequalities obtained.

We apply the Lemma used in I, choosing the interval $0 \leqq \phi \leqq 2 \pi$ as the circle $\gamma$ : $|x|=1$, using $x=e^{i \phi}$ on $\gamma$. The functions $\left\{u_{n}(\phi)\right\}$ and $\left\{U_{n}(\phi)\right\}$ are taken (modifying the argument of I, pp. 162-3) simply as

$$
u_{n}(\phi)=\frac{x^{n}}{(2 \pi)^{1 / 2}}, \quad U_{n}(\phi)=\frac{p_{n}(x)}{(2 \pi)^{1 / 2}} \quad(n=0,1,2, \cdots) .
$$

Thus we have immediatëly

$$
\begin{aligned}
& c_{n k}=\int_{0}^{2 \pi}\left(U_{n}-u_{n}\right) \bar{u}_{k} d \phi, \\
& \sum_{k=0}^{\infty} c_{n k} \bar{c}_{n k} \leqq \int_{0}^{2 \pi}\left(U_{n}-u_{n}\right)\left(\bar{U}_{n}-\bar{u}_{k}\right) d \phi \leqq \epsilon_{n}{ }^{2}, \\
& c_{n k}=\int_{0}^{2 \pi}\left(U_{n}-u_{n}\right) \bar{u}_{k} d \phi=\frac{1}{2 \pi i} \int_{\gamma}\left(p_{n}(x)-x^{n}\right) \frac{d x}{x^{k+1}} \\
& =\frac{1}{2 \pi i} \int_{\gamma^{\prime}}\left(p_{n}(x)-x^{n}\right) \frac{d x}{x^{k+1}}
\end{aligned}
$$




$$
\left|c_{n k}\right| \leqq \frac{\epsilon_{n}}{(1+\epsilon)^{k}}
$$

The function $V_{k}(\phi)$ of $\mathrm{I}$ is therefore given by the equation*

$$
V_{k}(\phi)=\sum_{i=0}^{\infty}\left(d_{k i}+\delta_{k i}\right) u_{i} .
$$

We have, however, the inequalities

$$
\begin{gathered}
\left|d_{k i}+c_{i k}\right| \leqq \frac{p_{i} p_{k}}{1-p}, \quad p_{i}^{2}=\sum_{j=0}^{\infty}\left|c_{i j}\right|^{2}, \quad p^{2}=\sum_{i, j=0}^{\infty}\left|c_{i j}\right|^{2}, \\
\sum_{i=0}^{\infty}\left|d_{k i}\right| \ll \sum_{i=0}^{\infty}\left|d_{k i}+c_{i k}\right|+\sum_{i=0}^{\infty}\left|c_{i k}\right| .
\end{gathered}
$$

We define the functions $P_{k}(x)$ so as to make the two following series identical:

$$
\begin{array}{ll}
\sum_{k=0}^{\infty} c_{k} p_{k}(x), & c_{k}=\int_{\gamma} F(x) P_{k}(x) d x, \\
\sum_{k=0}^{\infty} b_{k} U_{k}(\phi), & b_{k}=\int_{\gamma} F(x) \bar{V}_{k}(\phi) d \phi .
\end{array}
$$

That is, we set

$$
c_{k}=\frac{b_{k}}{(2 \pi)^{1 / 2}}, \quad P_{k}(x)=\frac{1}{(2 \pi)^{1 / 2}} \bar{V}_{k}(\phi) \frac{d \phi}{d x}
$$

We have of course

$$
x=e^{i \phi}, \quad d x=i e^{i \phi} d \phi, \quad \frac{d \phi}{d x}=\frac{1}{i x} .
$$

It follows, then, directly from (9) and (5) that the functions $P_{k}(x)$ are continuous for $|x| \geqq 1$, analytic for $|x|>1$, and zero at infinity. Moreover, if the series $\sum \epsilon_{k}$ is dominated by a convergent geometric series, then the functions $P_{k}(x)$ are analytic likewise for $|x|=1$. $\dagger$ In fact, the series (11) is also dominated by a convergent geometric series, by virtue of $(7)$ :

$$
p_{i} \leqq \epsilon_{i}
$$

* See Walsh; these Transactions, vol. 22 (1921), p. 234, where the Lemma used in I is proved, and inequalities (10) and (11) likewise derived.

† The writer withdraws the statement in I, pp. 159, 163, that the functions $P_{k}(x)$ are analytic on $\gamma$, when the $\epsilon_{k}$ are not further restricted. Thus in the proof of $I$, Theorem $I$, we choose the $\epsilon_{k}$ so that the series $\sum \epsilon_{k}$ is dominated by a convergent geometric series. 
and by virtue of (8). Then the series (9), when conjugate complex quantities are taken, is a Laurent series whose coefficients are dominated by a convergent geometric series, so $\bar{V}_{k}(x)$, and hence also $P_{k}(x)$, is analytic for $|x|=1$.

3. Development of continuous functions on $\gamma$. If the function $F(x)$ is continuous for $|x| \leqq 1$ and analytic for $|x|<1$, then the Taylor development of $F(x)$ about the origin converges, when summed by the method of Cesàro, uniformly for $|x| \leqq 1$ to the value $F(x)$. In fact the Taylor development is on $\gamma$ precisely the Fourier development of $F(x)$, which when summed as described converges uniformly on $\gamma$ to the value $F(x)$, hence uniformly on and within $\gamma$ to the value $F(x)$. The Taylor series itself converges for $|x|<1$, by the usual inequalities for the coefficients of a power series, and hence converges to the value $F(x)$, because in case of a convergent series the sum assigned by the Cesàro summation process is the sum of the series.

Application of this remark yields, if we remember that series (3) and (4) have essentially the same convergence properties in the entire closed region $|x| \leqq 1$,

THEOREM II. If $F(x)$ is continuous for $|x| \leqq 1$ and analytic for $|x|<1$, then the series (4) converges uniformly to the sum $F(x)$ in any closed region $|x| \leqq\left|x_{0}\right|<1$, and the sequence formed from (4) by the Cesàro summation method converges uniformly for $|x| \leqq 1$, to the sum $F(x)$.

We turn now from the consideration of series (4) arising from functions $F(x)$ given on $\gamma$, to the consideration of series of the form

$$
\sum_{k=0}^{\infty} g_{k} p_{k}(x)
$$

with arbitrary coefficients $g_{k}$.

4. Convergence of arbitrary series (13). If no further restriction is placed on the quantities $\epsilon_{k}$ than in Theorem I, it is not true that the convergence of (13) for $x=x_{0}$ enables us to conclude the convergence of (13) for all values of $x$ such that $|x|<\left|x_{0}\right|$. Let us set, in fact,

$$
p_{0}(x)=1, \quad p_{k}(x)=x^{k}-\delta^{k}, \quad k>0,
$$

where $\delta$ is positive and so small that for $\epsilon_{0}=0, \epsilon_{k}=\delta^{k}, k>0$, the required conditions on $\epsilon_{k}$ are fulfilled. Then every series (13) converges for $x=\delta$, yet need not converge for every $x$ such that $|x|<\delta$. Indeed, under this same definition for $p_{k}(x)$, every series

$$
\sum_{k=0}^{\infty} g_{2^{k} p_{2^{k}}(x)}
$$


converges whenever $x=\omega \delta, \omega^{2^{n}}=1, n$ being integral. This series converges therefore on a point set everywhere dense on the circle $|x|=\delta$, yet does not necessarily converge for $x=0$.

Under suitable restrictions on the $\epsilon_{k}$ we can prove the result for series (13) which is analogous to the well known result for Taylor's series:

Theorem III. If the series (13) converges for $x=x_{0}$, where $\left|x_{0}\right| \leqq 1+\epsilon$, and if the series $\sum_{k=0}^{\infty} \epsilon_{k} t^{k}$ converges for every (finite) value of $t$, then the series (13) converges for all values of $x$ such that $|x|<\left|x_{0}\right|$, and the convergence is uniform for all values of $x$ such that $|x| \leqq\left|x_{1}\right|<\left|x_{0}\right|$.

We naturally assume $x_{0} \neq 0$; the contrary case is without content. We prove actually a stronger theorem than that stated, for we use not the convergence of (13) for $x=x_{0}$ but merely the boundedness of the terms of the series.

The inequality

$$
\left|p_{k}\left(x_{0}\right)-x_{0}^{k}\right| \leqq \epsilon_{k}
$$

gives at once the double inequality

$$
1-\frac{\epsilon_{k}}{\left|x_{0^{k}}\right|} \leqq \frac{\left|p_{k}\left(x_{0}\right)\right|}{\left|x_{0}^{k}\right|} \leqq 1+\frac{\epsilon_{k}}{\left|x_{0}^{k}\right|} .
$$

But we have $\lim _{k \rightarrow \infty} \epsilon_{k} /\left|x_{0}\right|=0$, and hence $\lim _{k \rightarrow \infty}\left|p_{k}\left(x_{0}\right)\right| /\left|x_{0}\right|=1$. Therefore if the quantities $g_{k} p_{k}\left(x_{0}\right)$ are uniformly bounded, so also are the quantities $g_{k} x_{0}$, and conversely. From the boundedness of the $g_{k} x_{0}$ :

follows the inequality

$$
\left|g_{k} x_{0}^{k}\right|<M \text {, }
$$

$$
\left|g_{k}\right|<\frac{M}{\left|x_{0}^{k}\right|}
$$

We now make use of the inequality

or

$$
\left|p_{k}(x)\right| \leqq|x|^{k}+\epsilon_{k},
$$

$$
\sum_{k=0}^{\infty}\left|g_{k} p_{k}(x)\right| \ll \sum_{k=0}^{\infty}\left|g_{k}\right| \cdot|x|^{k}+\sum_{k=0}^{\infty}\left|g_{k}\right| \epsilon_{k} .
$$

The first series on the right converges uniformly for $|x| \leqq\left|x_{1}\right|$, since the individual terms of that series are bounded for $x=x_{0}$. The second series on the right, which does not contain $x$, converges. Hence the series on the left converges uniformly for $|x| \leqq\left|x_{1}\right|$, and Theorem III is established.

The argument just given includes practically a proof of the fact that for points $x$ such that $|x| \leqq 1+\epsilon$, the two series 


$$
\sum_{k=0}^{\infty} g_{k} x^{k} \text { and } \sum_{k=0}^{\infty} g_{k} p_{k}(x)
$$

have the same points of convergence, of absolute convergence, of divergence, of summability, and the same regions (or point sets) of uniform convergence. The only exception here occurs if the Taylor series converges only at the point $x=0$. For if there exists a single value of $x$, say $x_{0} \neq 0$, of the kind considered so that either of these two series converges, we have

$$
\left|g_{k}\right|<\frac{M}{\left|x_{0}^{k}\right|} .
$$

It follows that for all values of $x,|x| \leqq 1+\epsilon$, we have uniformly

$$
\left|g_{k} p_{k}(x)-g_{k} x^{k}\right| \leqq \frac{M \epsilon_{k}}{\left|x_{0}{ }^{k}\right|} .
$$

The series $\sum_{k=0}^{\infty} M \epsilon_{k} /\left|x_{0}{ }^{k}\right|$ converges and does not contain $x$, so the statement is proved.

There is no exceptional case here, even if the Taylor series converges only for $x=0$, provided $p_{k}(0)=0, k=1,2, \cdots$; compare Theorems VII and IX below.

We can state now two interesting results of this discussion; the first result gives the radius of convergence in terms of the coefficients. Here and in the remainder of the paper we assume, unless otherwise stated, the series $\sum \epsilon_{k} t^{k}$ to converge for all values of $t$.

TheOREM IV. If we set

$$
\varlimsup_{k \rightarrow \infty}\left|g_{k}\right|^{1 / k}=\frac{1}{\rho},
$$

then if $\rho \leqq 1+\epsilon$, series (13) converges for $|x|<\rho$ and diverges for $1+\epsilon>|x|>\rho$; if $\rho>1+\epsilon$, series (13) converges for $|x| \leqq 1+\epsilon$.

The generalized theorem of Abel yields its analogue for the series (13):

THEOREM V. If the series (13) converges for the value $x=x_{1}$, where $\left|x_{1}\right|$ $\leqq 1+\epsilon$, then this series converges uniformly in the closed region bounded by two arbitrary line segments terminating at the point $x_{1}$ and by an arc of the circle $|x|=\left|x_{2}\right|<\left|x_{1}\right|$.

If (13) converges uniformly on an arc $x_{1} x_{2}$ of the circle $|x|=\left|x_{1}\right|$, then this series converges uniformly in the closed region bounded by this circular arc, by two arbitrary line segments lying in the region $|x| \leqq\left|x_{1}\right|$ and terminated respectively by $x_{1}$ and $x_{2}$, and by an arc of a circle $|x|=\left|x_{3}\right|<\left|x_{1}\right|$. 
5. Analogue of Tauber's theorem. For series (13) we can give likewise a converse (not exact) of Abel's Theorem:

TheOREM VI. If the series (13) is such that $\lim _{k \rightarrow \infty} g_{k} / k=0$, and if for radial approach* to the point $x_{1}$ on $\gamma$ we have

$$
\lim _{x \rightarrow x_{1}} f(x)=g,
$$

where $f(x)$ denotes the value of the (convergent for $|x|<1$ ) series (13), then we have also

$$
\sum_{k=0}^{\infty} g_{k} p_{k}\left(x_{1}\right)=g .
$$

If we define the numbers $b_{k}$ by the relations

and then set

$$
b_{k}=(2 \pi)^{1 / 2} g_{k}
$$

$$
a_{k}=b_{k}+c_{0 k} b_{0}+c_{1 k} b_{1}+c_{2 k} b_{2}+\cdots \quad(k=0,1,2, \cdots),
$$

we find by the use of the Schwarz inequality in conjunction with (8),

$$
\left|a_{k}-b_{k}\right| \leqq \frac{\left(\sum_{i=0}^{\infty}\left|b_{i}\right|^{2}\right)^{1 / 2}}{(1+\epsilon)^{k}}
$$

By our hypothesis on the $g_{k}$, the series $\sum_{i=0}^{\infty}\left|b_{i}\right|^{2}$ converges. The $a_{k}$ defined by (14) are such that $\lim _{k \rightarrow \infty} a_{k} / k=0$, by (15). These numbers $a_{k}$ are such that $\sum_{k=0}^{\infty}\left|a_{k}\right|^{2}$ converges, hence, by the Riesz-Fischer theorem, there exists a function $F(x)$ defined on $\gamma$, integrable and with an integrable square, whose coefficients with respect to the normal orthogonal system $\left\{u_{k}\right\}$ used in $\$ 2$ are the numbers $a_{k}$. The numbers $b_{k}$, subjected to the condition that $\sum_{k=0}^{\infty}\left|b_{k}\right|^{2}$ should converge, are uniquely determined by (14), $\dagger$ and hence the numbers $g_{k}=b_{k} /(2 \pi)^{1 / 2}$ are the coefficients of $F(x)$ in its expansion (4). Thus (3) and (13) have essentially the same convergence properties in and on $\boldsymbol{\gamma}$

By Tauber's theorem, $\ddagger$ we have, since $\lim _{k \rightarrow \infty} a_{k} / k=0$,

$$
\sum_{k=0}^{\infty} a_{k} x_{i}^{k}=g
$$

The result holds also for approach in various other ways. See for example Landau, Ergebnisse der Funktionentheorie, Berlin, 1916, Kap. III. Compare our application of Theorem VI in Theorem IX.

t See the reference in $\$ 2$ to the proof of the Lemma used in I.

$\ddagger$ See Landau, loc. cit. 
and thus we have as well

$$
\sum_{k=0}^{\infty} g_{k} p_{k}\left(x_{1}\right)=g,
$$

and the theorem is established.

Many other results similar to Theorem VI, analogues of results for Taylor's series, might be established. We choose, however, to treat the equivalence of series (3) and (4) on circles other than $\gamma$.

6. Properties of series on circles other than $\gamma$. We prove the following theorem:

TheOREM VII. If $p_{k}(x)$ has at least a $k$-fold root at the origin, then an arbitrary function $F(x)$ integrable and with an integrable square on the circle $\Gamma:|x|=\mu<1+\epsilon$ can be formally expanded in a series of type (4), where the coefficients are found by integration over $\Gamma$. This series (4) and the Taylor development (formal) of $F(x)$ have on and within the circle $\Gamma$ the same convergence properties, in the sense that their term-by-term difference converges absolutely and uniformly on $\Gamma$ and in its interior to the sum zero.

We perform the substitution $z=x / \mu, x=\mu z$, so as to apply Theorem I directly to the unit circle in the $z$-plane. We require for application of Theorem I the inequality

$$
\left|\frac{p_{k}(\mu z)}{\mu^{k}}-z^{k}\right| \leqq \epsilon_{k} \text { for all }|z| \leqq 1+\epsilon^{\prime}, \quad \epsilon^{\prime}>0 .
$$

Expansion of the function $F(\mu z)$ on the circle $|z|=1$ in terms of the functions $p_{k}(\mu z) / \mu^{k}$, which approximate to the functions $z^{k}$, will yield of course a formal expansion of $F(x)$ on the circle $\Gamma$ in terms of the functions $p_{k}(x)$. The Taylor expansion of $F(\mu z)$, a power series in $z$, transforms into a Taylor expansion of $F(x)$, a power series in $x$.

Our original inequality

$$
\left|p_{k}(x)-x^{k}\right| \leqq \epsilon_{k},|x| \leqq 1+\epsilon,
$$

may be written

$$
\left|\frac{p_{k}(x)}{x^{k}}-1\right| \leqq \frac{\epsilon_{k}}{\left|x^{k}\right|}, \quad x \neq 0 .
$$

But the function $\left(p_{k}(x) / x^{k}\right)-1$ is analytic without exception for $|x| \leqq 1+\epsilon$, when properly defined for $x=0$, and its greatest absolute value in that closed region is taken on for $|x|=1+\epsilon$. Thus we have

$$
\left|\frac{p_{k}(x)}{x^{k}}-1\right| \leqq \frac{\epsilon_{k}}{(1+\epsilon)^{k}}, \quad|x| \leqq 1+\epsilon .
$$


Transformation to the $z$-plane gives the equivalent inequalities

$$
\begin{aligned}
& \left|\frac{p_{k}(\mu z)}{\mu^{k} z^{k}}-1\right| \leqq \frac{\epsilon_{k}}{(1+\epsilon)^{k}}, \quad \mu|z| \leqq 1+\epsilon, \\
& \left|\frac{p_{k}(\mu z)}{\mu^{k}}-z^{k}\right| \leqq \frac{\epsilon_{k}\left|z^{k}\right|}{(1+\epsilon)^{k}}, \quad \mu|z| \leqq 1+\epsilon .
\end{aligned}
$$

The right-hand member of (17) is not greater than $\epsilon_{k}$ provided we restrict $z$ as follows:

$$
|z| \leqq \frac{1+\epsilon}{\mu} \text { if } \mu>1, \quad|z| \leqq 1+\epsilon \text { if } \mu \leqq 1 .
$$

The upper limits of $z$ in (18) are both greater than unity, so (17) yields directly (16), and Theorem VII is completely established.

The proof of Theorem VII has not assumed any restriction on the quantities $\epsilon_{k}$ beyond that of Theorem I. In fact, the condition that $p_{k}(x)$ should have at least a $k$-fold root at the origin is a very favorable one with reference to successive approximations and equivalence of expansions. With the conditions imposed, the requirements on the $\epsilon_{k}$ of Theorem I can be considerably lightened; we do not, however, carry out the details here.

We remark, too, that a result similar to Theorem VII is readily proved under the assumption that a convergent geometric series dominates the series $\sum \epsilon_{k}$, without the assumption that $p_{k}(x)$ has at least a $k$-fold root at the origin; but here there is in general a lower limit greater than zero on the radius $\mu$ of the circle $\Gamma$. We omit the proof of this remark.

The following theorem is by no means the most general result of its kind that can be easily established:

TheOREM VIII. If $p_{k}(x)$ has at least a $k$-fold root at the origin, and if the series $\sum \epsilon_{k} t^{k}$ converges for every $t$, then the expansion of type (4) of any function $F(x)$ analytic at the origin is unique. The functions $P_{k}(x)$ of Theorem $I$ are analytic over the entire plane except at the origin.

If $F(x)$ is analytic on and within $\gamma$, there cannot exist two distinct expansions of $F(x)$ of the form

$$
F(x)=\sum_{k=0}^{\infty} c_{k} p_{k}(x), \quad F(x)=\sum_{k=0}^{\infty} g_{k} p_{k}(x)
$$

both of which converge uniformly on $\gamma$. For multiplication of these series through by $P_{k}(x) d x$ and integration term by term over $\gamma$ gives by (2) the equality of $c_{k}$ and $g_{k}$. 
We return to the more general situation of Theorem VIII. If two series of the form (19) both converge at even a single point for which $|x| \leqq 1+\epsilon$, they converge uniformly on and within some circle $\Gamma^{\prime}$ whose center is the origin. By the remark just made concerning uniqueness of expansions, and by the proof of Theorem VII, the two expansions are identical if they represent the same function on any circle whatever whose center is the origin. If the two series represent $F(x)$ in a region lying interior to the circle $|x|=1+\epsilon$, they both represent that function throughout their entire regions of convergence interior to the circle $|x|=1+\epsilon$.

The analyticity of the functions $P_{k}(x)$ of Theorem I over the entire plane except at the origin follows from (9) and (11) as used in \$2, with the new properties of the series $\sum \epsilon_{k}$. Compare also Theorem IXa, which does not use those new properties. Under the present hypothesis, then, the integrals which appear in (4) can be taken over any rectifiable Jordan curve which lies interior to $\gamma$ and in whose interior the origin lies, provided the function $F(x)$ is analytic for $|x| \leqq 1$. The functions $P_{k}(x)$ which arise in Theorem I for the circle $\gamma$, and the functions $P_{k}(x)$ which arise in the proof of Theorem VII by application of Theorem $I$ to the transform in the $z$-plane of the circle $\Gamma$ are identical; this can be verified by making the change of variable in all the formulas involved.

\section{B. SERIES OF POLYNomials}

7. Application of results of A. We now apply Theorem I and the theorems which have just been proved in connection with it, deriving results as in I (p. 163 et seq.) for expansions of arbitrary functions in terms of polynomials. We choose the quantities $\epsilon_{k}$ to satisfy the requirements of Theorem I and also so that $\sum \epsilon_{k} t^{k}$ converges for every $t .^{*}$ The polynomial $p_{k}(z)$ is to be chosen so as to have a $k$-fold root at the origin; this choice is possible; compare I, p. 164, or Theorem X below. Then we have

THEOREM IX. In the plane of the complex variable $z$ let $C$ be a simple closed finite analytic curve $\dagger$ which includes in its interior the origin. Then

* See also the condition of I, p. 164, and its application in the proof of Theorem IXa.

$\dagger$ That is to say, a curve whose points can be put into one-to-one (regular-) analytic correspondence with the points of a circle. It is then a classical theorem in the study of conformal mapping that the region interior to $C$ can be mapped on the interior of a circle so that the mapping is one-to-one and conformal in the closed regions considered, therefore one-to-one and conformal in larger regions including those closed regions in their interiors. See Picard, Traité d' A nalyse, II, Paris, 1893, pp. 272, 276, or Bieberbach, Einführung in die konforme Abbildung, Berlin, 1913, p. 120.

From this theorem it follows at once, in the notation of I or of Theorem IX, that for points $z_{1}$ and $z_{2}$ on $C$, the quotients $\left(z_{1}-z_{2}\right) /\left(\phi\left(z_{1}\right)-\phi\left(z_{2}\right)\right)$ and $\left(\phi\left(z_{1}\right)-\phi\left(z_{2}\right)\right) /\left(z_{1}-z_{2}\right)$ are uniformly bounded. Hence a function which satisfies a Lipschitz condition on $C$ corresponds to a function which satisfies a Lipschitz condition on the circle $\gamma$, and conversely. 
the interior of $C$ can be mapped one-to-one and conformally on the interior of the unit circle $\gamma$ in the $x$-plane by some transformation $x=\phi(z), z=\psi(x)$, where $\phi(0)=0$, and the transformation will be one-to-one and conformal for the mapping of the closed interior of $C_{1+e}$, an analytic Jordan curve in whose interior $C$ lies, onto the closed interior of the circle $|x|=1+\epsilon, \epsilon>0$. In general we denote by $C_{\rho}$ the transform of the circle $|x|=\rho$, where $0<\rho<1+\epsilon$.

Then there exists a set $\left\{p_{k}(z)\right\}$ of polynomials in $z$ and a set of functions $\left\{s_{k}(z)\right\}$ analytic at every point of the extended plane except the origin, zero at infinity, and such that

$$
\int_{C_{\rho}} s_{k}(z) p_{i}(z) d z=\left\{\begin{array}{l}
0, i \neq k, \\
1, i=k .
\end{array}\right.
$$

If the function $F(z)$ is analytic interior to $C_{p}$, continuous in the corresponding closed region, and satisfies a Lipschitz condition on $C_{\rho}$, then the series

$$
\sum_{k=0}^{\infty} a_{k} p_{i}(z), \quad a_{k}=\int_{C_{\rho}} F(z) s_{k}(z) d z,
$$

converges uniformly in this closed region to the value $F(z)$. If $F(z)$ is required merely to be analytic interior to $C_{p}$ and continuous in the corresponding closed region, then (20) converges uniformly to the value $F(z)$ interior to an arbitrary curve $C_{\rho^{\prime}}, \rho^{\prime}<\rho$, and when summed by the method of Cesaro, (20) converges uniformly to the value $F(z)$ in the closed region bounded by $C_{\rho}$.

If $F(z)$ is an arbitrary function defined on $C_{p}$, integrable and with an integrable square, and if the condition*

$$
\int_{C_{\rho}} F(z) z^{k} d z=0 \quad(k=0,1,2, \cdots),
$$

is satisfied, then the two series

$$
\sum_{k=0}^{\infty} a_{k}^{\prime} x^{k}, \quad a_{k}^{\prime}=\frac{1}{2 \pi i} \int_{|x|=\rho} F[\psi(x)] x^{-k-1} d x,
$$

and (20) transformed by $z=\psi(x)$ have essentially the same convergence properties on and within the circle $|x|=\rho$, in the sense that their term-by-term difference converges absolutely and uniformly to the sum zero for $|x| \leqq \rho$.

* No condition is necessary here if we use

instead of (20).

$$
a=\int_{|x|-\rho} F[\psi(x)] P_{k}(x) d x
$$


An arbitrary series of the form

$$
\sum_{k=0}^{\infty} g_{k} p_{k}(x)
$$

which converges for a single point $z$ on $C_{\rho}$, converges uniformly interior to $C_{\rho^{\prime}}$, if $\rho^{\prime}<\rho$. If (21) diverges for a point $z$ on $C_{\rho}$ that series diverges for all points $z$ exterior to $C_{\rho}$ and interior to $C_{1+e}$. If in general we set

$$
\limsup _{k \rightarrow \infty}\left|g_{k}\right|^{1 / k}=\frac{1}{\rho}
$$

then if $\rho \leqq 1+\epsilon$, series (21) converges for $z$ interior to $C_{\rho}$ and diverges for $z$ exterior to $C_{\rho}$ but interior to $C_{1+e} ;$ if $\rho>1+\epsilon$, series (21) converges for $z$ on or interior to $C_{1+e}$. If $0<\rho<1+\epsilon$, some singular point of the function represented by the series lies on the curve $C_{\rho}$.

If (21) converges for the value $z=z_{1}$ on $C_{\rho}$, then this series converges uniformly in the closed region bounded by two arbitrary line segments terminating in $\mathbf{z}_{1}$, and by an arc of a curve $C_{\rho^{\prime}}$, where $\rho^{\prime}<\rho$. If (21) converges uniformly on an arc $z_{1} z_{2}$ of the curve $C_{p}$ then this series converges uniformly in the closed region bounded by that arc, by two arbitrary line segments whose interiors are interior to $C_{\rho}$ and which are terminated respectively by $z_{1}$ and $z_{2}$, and by an arc of the curve $C_{\rho^{\prime}}$, where $\rho^{\prime}<\rho$.

If (21) is such that $\lim _{k \rightarrow \infty} g_{k}\left(k \rho^{k}\right)^{-1}=0$, and if for approach along the normal ${ }^{*}$ to $C_{p}$ to the point $z_{1}$ on $C_{p}$ we have

$$
\lim _{s \rightarrow s_{1}} f(z)=g,
$$

where $f(z)$ denotes the value of the (convergent for $z$ interior to $C_{\rho}$ ) series (21), then we have also

$$
\sum_{k=0}^{\infty} g_{k} p_{k}\left(z_{1}\right)=g \text {. }
$$

An arbitrary series (21), convergent for a single value of $z$ interior to $C_{1+1}$ and not the origin, is the unique expansion of form (20) of some function $F(z)$ analytic on and within some curve $C_{p}$.

The only part of this theorem not a direct result of our previous theorems is the fact that $s_{k}(z)$ is analytic over the entire $z$-plane except at the origin. This should give the reader no difficulty, using Theorem VIII and the method of I, p. 165 ; compare also $\S 9$.

- A more general theorem might easily be announced; see the footnote to Theorem VI. Here we do not apply Theorem VI directly, but the more general theorem suggested in connection with Theorem VI. 
It will be notic ${ }^{-1}$ that Theorem IX does not mention convergence of the series (20) or (21) outside of $C_{1+e}$. The reason for this omission will become clearer after we have proved a general theorem on approximation.

8. A general theorem on approximation. We prove a much more general theorem than necessary for our immediate purposes:*

TheOREM X. If the function $f(z)$, defined on the bounded point set $S$, can be approximated on that point set as closely as desired by a polynomial in $z$, and if there be given any $p$ points $z_{1}, z_{2}, \cdots, z_{p}$ of $S$ together with an arbitrary $\epsilon>0$, then there exists a polynomial $p(z)$ such that

and

$$
|p(z)-f(z)| \leqq \epsilon, \quad z \text { on } S,
$$

$$
p\left(z_{i}\right)=f\left(z_{i}\right) \quad(i=1,2, \cdots, p) .
$$

We prove Theorem $\mathrm{X}$ by means of Lagrange's Interpolation Formula, and find it convenient first to prove the following

Lemma. If $z_{1}, z_{2}, \cdots, z_{p}, R$ are considered fixed, if we have

$$
\left|G_{k}\right| \leqq \eta \quad(k=1,2, \cdots, p),
$$

and if $G(z)$ denotes the polynomial defined by Lagrange's Interpolation Formula which takes on the values $G_{k}$ in the points $z_{k}, k=1,2, \cdots, p$, then there exists a constant $M$ independent of $\eta$ so that we have

$$
|G(z)| \leqq M \eta \text { for all } z,|z| \leqq R .
$$

For simplicity we take $R$ so large that $\left|z_{k}\right| \leqq R, k=1,2, \cdots, p$. The Lagrange Formula is

$$
G(z)=\sum_{p=1}^{p} G_{v} \frac{\left(z-z_{1}\right) \cdots\left(z-z_{p-1}\right)\left(z-z_{p+1}\right) \cdots\left(z-z_{p}\right)}{\left(z_{p}-z_{1}\right) \cdots\left(z_{p}-z_{v-1}\right)\left(z_{p}-z_{v+1}\right) \cdots\left(z_{p}-z_{p}\right)},
$$

so the Lemma is obvious if we merely set

$$
M=\sum_{\nu=1}^{p} \frac{(2 R)^{p-1}}{\left|z_{\nu}-z_{1}\right| \cdots\left|z_{p}-z_{p-1}\right| \cdot\left|z_{p}-z_{p+1}\right| \cdots\left|z_{p}-z_{p}\right|} .
$$

\footnotetext{
* It seems inconceivable that this theorem is not already in the literature, but the writer has been unable to find a reference to it. The corresponding theorem for approximation by trigonometric polynomials is given by D. Jackson, Bulletin of the American Mathematical Society, vol. 32 (1926), pp. 259-262.

Theorem $\mathrm{X}$ holds of course for approximation of real functions by means of real polynomials, and can be extended (1) by requiring the agreement of certain derivatives of the approximating polynomial with the corresponding derivatives of the given function, (2) by assigning as the values of the polynomial (and derivatives) not the exact but values near to the values of the function (and derivatives), (3) by noticing that for points $z_{k}$ off $S$ arbitrary values $f\left(z_{k}\right)$ may be assigned.
} 
Theorem $\mathrm{X}$ follows easily now. Choose $R$ so large that all points of $S$ lie in the circle $|z| \leqq R$. Choose a polynomial $q(z)$ (which exists by hypothesis) so that we have

$$
|q(z)-f(z)| \leqq \frac{\epsilon}{1+M}, \quad z \text { on } S .
$$

For the polynomial $G(z)$ we assign the values

$$
G\left(z_{k}\right)=q\left(z_{k}\right)-\left(f z_{k}\right) \quad(k=1,2, \cdots, p),
$$

so that we have by the Lemma

Then the polynomial

$$
|G(z)| \leqq \frac{M \epsilon}{1+M}, \quad z \text { on } S .
$$

$$
p(z)=q(z)-G(z)
$$

satisfies all the requirements of Theorem $X$.

The polynomials $p_{k}(z)$ of Theorem IX are polynomials which uniformly approximate to the functions $[\phi(z)]^{k}$ respectively on and within $C_{1+c .}$ By a classical theorem due to Runge, these polynomials may be subjected to the auxiliary condition of uniformly approximating other analytic functions - let us say constants - in arbitrary non-intersecting closed Jordan regions outside of $C_{1+e}$. In particular we may by Theorem $\mathrm{X}$ require that the polynomials $p_{k}(z)$ shall actually take on arbitrarily preassigned values at an arbitrary number of points exterior to $C_{1+e}$. Thus we may choose (1) the value zero for the points $z_{1}, z_{2}, \cdots, z_{p}$ (independent of $k$ ), in which case all series of the form (21) converge at those points, or we may choose (2)

$$
p_{k}\left(z_{i}\right)=k ! \quad(i=1,2, \cdots, p ; \quad k=1,2,3, \cdots),
$$

in which case no series (21) not convergent throughout the interior of $C_{1+c}$ converges at the points $z_{i}$. It is because of this difference in behavior that Theorem IX omits mention of the convergence or divergence of (21) outside of $C_{1+e}$.

9. Further properties of expansions. One interesting property of series (20) has not yet been mentioned, which brings out still more clearly the analogy with Taylor's series:

ThEOREM IXa. The coefficients $a_{k}$ in (20) can be written in the form

$$
a_{k}=A_{0}{ }^{(k)} F(0)+A_{1}{ }^{(k)} F^{\prime}(0)+\cdots+A_{k}{ }^{(k)} F^{(k)}(0),
$$

where $A_{i}{ }^{(k)}$ is a constant independent of $F(z)$, and where $F^{(i)}(0)$ indicates the ith derivative of $F(z)$ at the origin. 
Differentiation of (20), with insertion of the value $z=0$, yields

$$
\begin{aligned}
F(0) & =a_{0} p_{0}(0), & & \\
F^{\prime}(0) & = & & a_{1} p_{1}^{\prime}(0), \\
F^{\prime \prime}(0) & = & & a_{1} p_{1}^{\prime \prime}(0)+a_{2} p_{2}^{\prime \prime}(0), \\
F^{\prime \prime \prime}(0) & = & & a_{1} p_{1}^{\prime \prime \prime}(0)+a_{2} p_{2}^{\prime \prime \prime}(0)+a_{3} p_{3}^{\prime \prime \prime}(0),
\end{aligned}
$$

Here we use the fact that $p_{0}(z)$ is constant, and that $p_{k}(z)$ has at least a $k$-fold root at the origin and hence (I, p. 164), having precisely $k$ roots interior to $C_{1+e}$, has precisely a $k$-fold root at the origin. This system of equations is therefore such that $p_{k}{ }^{(k)}(0)$ is always different from zero, $k=0,1,2, \cdots$, and hence the system can be solved for the coefficients $a_{k}$ linearly in terms of the $F^{(i)}(0)$.

As an application of Theorem IXa, it may be noticed that $s_{k}(z)$ can be written in the form

$$
S_{0}(z)=\frac{B_{1}{ }^{(0)}}{z}, S_{k}(z)=\frac{B_{2}^{(k)}}{z^{2}}+\frac{B_{3}{ }^{(k)}}{z^{3}}+\cdots+\frac{B_{k+1}^{(k)}}{z^{k+1}}, k>0 ;
$$

this follows directly from the integral formula for the derivatives of $F(z)$.

One may consider in some detail the expansion of an arbitrary function $\Phi(z)$, analytic on and interior to $C$, in terms not of the polynomials $p_{k}(z)$ but in terms of their derivatives $p_{k}^{\prime}(z)$. Let $F(z)$ be any integral of $\Phi(z)$, so that we have for $z$ on and interior to $C$,

$$
\begin{aligned}
& F(z)=a_{0} p_{0}(z)+a_{1} p_{1}(z)+a_{2} p_{2}(z)+\cdots, \quad a_{k}=\int_{C} F(z) s_{k}(z) d z, \\
& F^{\prime}(z)=\Phi(z)=a_{1} p_{1}^{\prime}(z)+a_{2} p_{2}^{\prime}(z)+\cdots .
\end{aligned}
$$

The term $a_{0} p_{0}^{\prime}(z)$ is here omitted, for $p_{0}(z)$ is a constant.

The integral

$$
\int_{C} s_{k}(z) d z, \quad k>0,
$$

is equal to zero, for this integral may be written

$$
\frac{1}{p_{0}(z)} \int_{C} s_{k}(z) p_{0}(z) d z
$$

known to vanish by Theorem IX. Hence the indefinite integral $\sigma_{k}(z)$ of $s_{k}(z)$ is single-valued in and on $C$. 
Let us integrate

$$
a_{k}=\int_{C} F(z) s_{k}(z) d z, \quad k>0,
$$

by parts, $\int u d v=u v-\int v d u$, setting $u=F(z), d v=s_{k}(z) d z$. We find

$$
a_{k}=-\int_{C} \Phi(z) \sigma_{k}(z) d z
$$

As is to be expected, we find also by partial integration

$$
\int_{C} \sigma_{k}(z) p_{i}^{\prime}(z) d z=-\int_{C} s_{k}(z) p_{i}(z) d z=-\delta_{i k} .
$$

That is, there exists a set of functions $\left\{-\sigma_{k}(z)\right\}$ such that the two sets $\left\{p_{k}^{\prime}(z)\right\}$ and $\left\{-\sigma_{k}(z)\right\}$ are biorthogonal. An arbitrary function $\Phi(z)$ analytic on and within $C$ can be expanded in the series

$$
\Phi(z)=a_{1} p_{1}^{\prime}(z)+a_{2} p_{2}^{\prime}(z)+\cdots, \quad a_{k}=-\int_{C} \Phi(z) \sigma_{k}(z) d z,
$$

which converges uniformly on and within $C$.

Both this remark on the derived functions and Theorem IXa can be applied in the $x$-plane to the series (4) under the hypothesis of Theorem VIII.

10. Expansion of discontinuous functions. There are considered in I not merely series such as (20), but likewise series in polynomials $q_{k}(z)$ in the reciprocal of $z{ }^{*}$ These series are used in $I$ to expand arbitrary functions satisfying a Lipschitz condition on $C$. In order to study the expansion of discontinuous functions in such series, we investigate the function or functions represented by Cauchy's Integral

$$
F(z)=\frac{1}{2 \pi i} \int_{C} \frac{f(t) d t}{t-z},
$$

where the given function $f(t)$ is discontinuous. We shall suppose $C$ to be the same curve previously considered, although the discussion holds under much broader conditions.

A particularly simple kind of discontinuity, that of a finite jump, is typified by the function $f(t)=\log t$, where we choose as that branch of the

- We notice that the argument used in I, p. 166, to prove $b_{0}=0$ can be somewhat shortened. We choose, in fact, $q_{0}(z)=1, q_{k}(\infty)=0$ for $k>0$. Then in the expansion of $f_{2}(z)$ :

it is obvious that $b_{0}=0$ when $f_{2}(z)=0$ for $z=\infty$.

$$
f_{2}(z)=\sum_{k=0}^{\infty} b_{k} q_{k}(z)
$$


function $\log t$ the branch which is real and positive for the smallest real positive value of $t$ on $C$, say $t_{0}$. Consider in general the plane cut along the line $0 t_{n}$, and from $t_{0}$ to infinity along a curve exterior to $C$. 'The determination of the branch of $\log t$ considered is then made by the use of continuity in the cut plane. The function $f(t)$ is continuous on $C$ except for a finite jump at $t_{0}$ of magnitude $2 \pi i$.

We evaluate the integral

$$
F(z)=\frac{1}{2 \pi i} \int_{c} \frac{\log t}{t-z} d t
$$

by partial integration, $\int u d v=u v-\int v d u$, setting $u=\log t, d v=d t /(t-z)$. We find

$$
F(z)=\left.\frac{1}{2 \pi i} \log t \cdot \log (t-z)\right|_{C}-\frac{1}{2 \pi i} \int_{C} \frac{\log (t-z)}{t} d t .
$$

The first term in the right-hand member has the value $2 \pi i+\log t_{0}+\log$ $\left(t_{0}-z\right)$, or $\log \left(t_{0}-z\right)$, according as $z$ lies interior or exterior to $C$. The proper determination of $\log (t-z)$ is to be found by continuity, moving $t$ along $C$ until it coincides with $t_{0}$, then by moving $z$, not crossing the cut, until $z$ coincides with the origin. The second term in the right-hand member has a zero derivative with respect to $z$, if $z$ lies interior to $C$, as is seen by direct computation. The value of the integral, $z$ interior to $C$, is therefore a constant equal to the value for $z=0$ :

$$
-\frac{1}{2 \pi i} \int_{c} \frac{\log t}{t} d t=-\left.\frac{1}{4 \pi i} \log ^{2} t\right|_{c}=-\pi i-\log t_{0} .
$$

The value of this same integral

$$
-\frac{1}{2 \pi i} \int_{c} \frac{\log (t-z)}{t} d t
$$

when $z$ lies exterior to $C$ is, by Cauchy's Formula, $-\log (-z)$. We have finally, therefore,

$$
\begin{aligned}
& f_{1}(z) \equiv F(z)=\pi i+\log \left(t_{0}-z\right), \quad z \text { interior to } C, \\
& f_{2}(z) \equiv-F(z)=-\pi i-\log \left(t_{0}-z\right)+\log z, \quad z \text { exterior to } C .
\end{aligned}
$$

As a check we have $f(z)=f_{1}(z)+f_{2}(z)$ when $z$ lies on $C$ (except in case $z=t_{0}$, where the functions $f_{1}(z)$ and $f_{2}(z)$ are, strictly speaking, not defined), as we should have by the results of Plemelj. ${ }^{*}$ The function $f_{1}(z)$ is analytic on

* See I, p. 167. The validity of the equation $f(z)=f_{1}(z)+f_{2}(z)$ is dependent, provided $f(z)$ satisfies certain large conditions of integrability, merely on the behavior of the function $f(z)$ in the neighborhood of the point $z$ considered; the satisfaction of a Lipschitz condition in such a neighborhood is sufficient for the validity of the equation. 
and interior to $C$ except at the single point $t_{0}$; the function $f_{2}(z)$ is analytic on and exterior to $C$ except at $t_{0}$ and vanishes at infinity; both functions are integrable and have an integrable square on $C$. We notice too by direct computation

$$
\begin{array}{lr}
\int_{C} f_{1}(t) t^{n} d t=0 & (n=0,1,2, \cdots), \\
\int_{C} f_{2}(t) t^{n} d t=0 \quad(n=-1,-2,-3, \cdots),
\end{array}
$$

from which follow the formulas (notation of $I$ ) for the coefficients in the expansion of $f_{1}(t)$ and $f_{2}(t)$ :

$$
\begin{array}{ll}
\int_{C} f_{1}(t) t_{k}(t) d t=0 & (k=1,2,3, \cdots), \\
\int_{C} f_{2}(t) s_{k}(t) d t=0 & (k=0,1,2, \cdots) .
\end{array}
$$

We use here in proving (22) the fact that $t_{k}(t)$ is analytic on and within $C$, hence on $C$ can be expressed as a uniformly convergent series of polynomials in $t$; likewise $s_{k}(t)$ is analytic on and exterior to $C$, hence on $C$ can be expressed as a uniformly convergent series of polynomials in $1 / t$ each without constant term. Such series may be integrated term by term, even after multiplication by $f_{1}(z)$ or $f_{2}(z)$.

The development of $f_{1}(z)$ on $C$ in terms of the polynomials $p_{k}(z)$ has essentially the same convergence properties as the development of the function

$$
\pi i+\log \left[t_{0}-\psi(x)\right]
$$

in a Fourier series (which is precisely the same as the development of the function in a Taylor or Laurent series) on the unit circle $\gamma$ in the $x$-plane. The development of $f_{2}(z)$ on $C$ in the polynomials $q_{k}(z)$ has essentially the same convergence properties as the development of

$$
-\pi i-\log \left[t_{0}-\psi_{1}(x)\right]+\log \psi_{1}(x)
$$

in a Fourier (or Laurent) series on $\gamma$, where we may take the solutions $x=x_{0}$ of the two equations

$$
\psi(x)=t_{0}, \quad \psi_{1}(x)=t_{0}
$$

equal, ${ }^{*} \psi_{1}(x)$ being a mapping function for the exterior of $\gamma$ onto the exterior of $C$, with correspondence of the points at infinity. These two functions

- Rotation of axes does not alter the convergence properties of a Taylor or Fourier development. 
just considered in the $x$-plane are both integrable with an integrable square and on $\gamma$ possess continuous derivatives except at the point $x_{0}$. The developments of the two functions converge therefore to the values of the respective functions except at $x_{0}$, and uniformly except in the neighborhood of $x_{0}$. In the neighborhood of the point $x_{0}$ the term-by-term sum of the two developments converges like the Fourier development of

$$
\log \frac{t_{0}-\psi(x)}{t_{0}-\psi_{1}(x)}+\log \psi_{1}(x)
$$

The latter term contains the only discontinuity, a finite jump of magnitude $2 \pi i$. Gibbs's phenomenon therefore occurs in its characteristic form at this point $x_{0}$; the series converges to the value which is the arithmetic mean of the limits approached in the two directions on $\gamma$ at $x_{0}$ by the function developed.

The Fourier development of $f_{2}(t)$ transformed by either $t=\psi(x)$ or $t=\psi_{1}(x)$ but interpreted for the same values of $t$ has essentially the same convergence properties in the two cases.

The discussion we have given is not essentially dependent on the particular choice of $t_{0}$ made originally. We may therefore state

THEOREM XI. If the function $F(z)$ satisfies a Lipschitz condition on $C$, and if the function

$$
f(z)=F(z)+k_{1} \log z+k_{2} \log z+\cdots+k_{m} \log z,
$$

where each term $k_{i} \log z$ is continuous on $C$ except at a single point $z_{i}$ of $C$, $z_{i} \neq z_{k}(i \neq k), k_{k} \neq 0$, be developed in a series (2) as in I ( $\left.p .156\right)$, then the Fourier development of $f(z)$ on the unit circle $|x|=1$, where $z=\psi(x)$, and the series

$$
f(z)=a_{0} p_{0}(z)+\left[a_{1} p_{1}(z)+b_{1} q_{1}(z)\right]+\left[a_{2} p_{2}(z)+b_{2} q_{2}(z)\right]+\cdots
$$

have essentially the same convergence properties* on C. In particular (23) exhibits Gibbs's phenomenon at the points $z_{k}$ precisely as does a Fourier series. On any closed arc of $C$ containing no point $z_{k}$, the series

$$
a_{0} p_{0}(z)+a_{1} p_{1}(z)+a_{2} p_{2}(z)+\cdots
$$

* The statement that two series have the same convergence properties is used in two senses in the literature, to indicate (1) that their term-by-term difference converges uniformly to the sum zero, or (2) that their term-by-term difference converges absolutely and uniformly to the sum zero. The present writer has hitherto consistently used the second of these two meanings, but in the present case implies (1) instead of (2). The treatment given here considers uniform convergence but not absolute convergence. 
converges to the value $f_{1}(z)$ and the series

$$
b_{1} q_{1}(z)+b_{2} q_{2}(z)+\cdots
$$

converges uniformly to the value $f_{2}(z)$, where

$$
f_{1}(z) \equiv \frac{1}{2 \pi i} \int_{C} \frac{f(t) d t}{t-z}
$$

is analytic interior to $C$ and continuous in the corresponding closed region except at the points $z_{k}$, and

$$
f_{2}(z) \equiv \frac{1}{2 \pi i} \int_{C} \frac{f(t) d t}{t-z}
$$

is analytic exterior to $C$, vanishes at infinity, and is continuous in the corresponding closed region except at the points $z_{k}$. For $z=z_{k}$ the two series (24) and (25) diverge with infinite sum, whereas the series (23) converges and its sum is the arithmetic mean of the two limits approached by $f(z)$ as a moves in opposite senses on $C$ and approaches $z_{k}$. If an arbitrary neighborhood of each of the points $z_{k}$ is cut out of the closed interior of $C$, the series (24) converges uniformly to the value $f_{1}(z)$ in the remaining closed region. If an arbitrary neighborhood of each of the points $z_{k}$ is cut out of the closed exterior of $C$, the series (25) converges uniformly to the value $f_{2}(z)$ in the remaining closed region.

Theorem XI is proved under the hypothesis on the $\epsilon_{k}$ that $\sum \epsilon_{k} t^{k}$ converges for every $t$.

Actual formulas for $f_{1}(z)$ and $f_{2}(z)$ in terms of logarithms and the functions represented by the integral

$$
\frac{1}{2 \pi i} \int_{c} \frac{F(t) d t}{t-z}
$$

can easily be written down. Of course, any function which is smooth except for a finite number of finite jumps can be put into the form of $f(z)$ of this theorem.

The conclusion of Theorem XI naturally holds for the formal Laurent development of a discontinuous function of the kind considered, if the curve $C$ is a circle. In particular it is the divergence of the Taylor series for $\log (x-a)$ for $x=a$ that enables us to conclude the divergence of (24) and (25) for $z=z_{k}$.

\section{BOUNDARY VALUES OF AN ANALYTIC FUNCTION}

11. A condition for analyticity. We now take up the study of the 
boundary values of an analytic function, later for a multiply-connected region but first for a simply-connected region:*

THEOREM XII. If the function $f(z)$ is continuous on the analytic Jordan curve $C$, and if we have

$$
\int_{C} f(z) z^{n} d z=0 \quad(n=0,1,2, \cdots),
$$

then there exists a function $F(z)$ analytic interior to $C$, continuous in the closed region which consists of $C$ and its interior, and which coincides with $f(z)$ on $C$.

If the curve $C$ is the unit circle $|z|=1$, the theorem is surely true. In fact the formal Laurent development of $f(z)$ is of the form of a Taylor series, since by (26) the coefficients of the negative powers of $z$ vanish:

$$
f(z) \sim a_{0}+a_{1} z+a_{2} z^{2}+\cdots, \quad a_{n}=\frac{1}{2 \pi i} \int_{c} \frac{f(z)}{z^{n+1}} d z .
$$

This development is precisely the formal Fourier development of $f(z)$ for $0 \leqq \phi \leqq 2 \pi$ if we set $z=e^{i \phi}$. The Fourier development, when summed by the method of Cesàro, converges uniformly on $C$, since $f(z)$ is continuous. Each term of the corresponding sequence is analytic on and interior to $C$, hence the sum of the series is analytic interior to $C$, continuous in the corresponding closed region, and is equal to $f(z)$ on $C$. This completes the proof of the theorem when $C$ is the unit circle.

If $C$ is not the unit circle, we map the interior of $C$ onto the interior of the unit circle $\gamma$ in the $x$-plane, the transformation being as usual $x=\phi(z)$, $z=\psi(x)$. The function $[\phi(z)]^{n} \phi^{\prime}(z)$ is analytic in and on $C$, hence on $C$ can be (by Runge's theorem) uniformly expanded in a series of polynomials in $\mathrm{z:}$

$$
[\phi(z)]^{n} \phi^{\prime}(z)=\pi_{0}(z)+\pi_{1}(z)+\pi_{2}(z)+\cdots, n \geqq 0 .
$$

This series converges uniformly on $C$ even after multiplication term by term by the continuous function $f(z)$. Term-by-term integration of the new series thus formed yields, by virtue of (26),

$$
\int_{c} f(z)[\phi(z)]^{n} \phi^{\prime}(z) d z=0 \quad(n=0,1,2, \cdots) .
$$

- In connection with this problem and the conditions derived, see F. and M. Riesz, Comples Rendus du Congrès (1916) des Mathematiciens Scandinaves, Uppsala, 1920, pp. 27-44; Privaloff, L'Integrale de Cauchy, Saratow, 1919; Kakeya, Tohoku Mathematical Journal, vol. 5 (1914), pp. 40-44, as well as the references given in I, p. 167. 
We have, then,

$$
\int_{\gamma} f[\psi(x)] x^{n} d x=0 \quad(n=0,1,2, \cdots),
$$

so by the special case of the theorem already proved there exists a function analytic interior to $\gamma$, continuous in the corresponding closed region, and coinciding on $\gamma$ with the function $f[\psi(x)]$. Transformation by the formula $x=\phi(z)$ gives the required function in the $z$-plane.

Conditions (26), it may be remarked, are all independent of each other and none of them may be omitted. If all of those conditions except a finite number are satisfied, then there exists a function $F(z)$ and a polynomial $P(z)$ in $1 / z$ such that $F(z)$ is analytic interior to $C$, continuous in the corresponding closed region, and such that

$$
F(z)+P(z)=f(z), z \text { on } C .
$$

The polynomial $P(z)$ is uniquely determined if we require that it shall vanish at infinity; otherwise is uniquely determined only to within an additive constant.

An alternate statement for Theorem XII is

TheOREM XIIa. If the function $f(z)$ is continuous on the analytic Jordan curve $C$, and if we have

$$
\int_{C} f(z) \omega(z) d z=0
$$

for every function $\omega(z)$ analytic in the closed region* interior to $C$, then there exists a function $F(z)$ analytic interior to $C$, continuous in the closed region which consists of $C$ and its interior, and which coincides with $f(z)$ on $C$.

The equivalence of (26) and (27) is easy to show. If (27) holds, (26) is surely satisfied. If (26) holds, then an arbitrary function $\omega(z)$ of the kind considered in Theorem XIIa can be uniformly expanded, in the closed region consisting of $C$ and its interior, in a series of polynomials:

$$
\omega(z)=\pi_{0}(z)+\pi_{1}(z)+\pi_{2}(z)+\cdots .
$$

Term-by-term multiplication of this series by $f(z)$ and term-by-term integration yield, by means of (26), equation (27). Thus (27) is both a necessary and a sufficient condition for the existence of $F(z)$.

There is a similar statement for functions representing the boundary values of a function analytic at infinity:

* We have here an equivalent condition if $\omega(z)$ is required to be analytic merely interior to $C$ and continuous in the corresponding closed region. 
THEOREM XIII. Let the function $f(z)$ be continuous on the analytic Jordan curve $C$, in whose interior the origin lies; then the two equivalent conditions

$$
\begin{aligned}
& \int_{C} f(z) z^{k} d z=0 \quad(k=-1,-2,-3, \cdots) ; \\
& \int_{C} f(z) \omega(z) d z=0,
\end{aligned}
$$

for every function $\omega(z)$ analytic exterior to $C$ (also at infinity), continuous in the corresponding closed region, and zero at infinity-these two conditions are each necessary and sufficient that there should exist a function $F(z)$ zero at infinity, analytic exterior to $C$ (including the point at infinity), continuous in the corresponding closed region, and equal to $f(z)$ on $C$.

The proof of this theorem is easy and will be omitted.

If in condition (A) we omit $k=-1$, and in (B) require that $\omega(z)$ should have a double root at infinity, those two conditions remain equivalent. The conditions are then necessary and sufficient for the existence of $F(z)$, analytic exterior to $C$ (including the point at infinity), continuous in the corresponding closed region, and equal to $f(z)$ on $C$. We cannot say, however, that $F(z)$ vanishes at infinity.

It will be noticed that if the continuous function $f(z)$ satisfies (26) as well as (A) of Theorem XIII, the two functions defined interior and exterior to $C$ respectively are analytic in the neighborhood of $C$, hence analytic also on $C$. The function analytic exterior to $C$ vanishes at infinity, so $f(z)$ is identically zero.

12. Extension to multiply-connected regions. In extending Theorems XII and XIII to the case of regions bounded by several contours, we shall mention merely the analogue of condition (A) although the analogue of condition (B) is easily included.

Theorem XIV. If the analytic Jordan curve $C^{\prime}$ lies interior to the analytic Jordan curve $C$, if the origin lies interior to $C^{\prime}$, and if the functions $f_{1}(z)$ and $f_{2}(z)$ continuous on $C$ and $C^{\prime}$ respectively satisfy the conditions

$$
\int_{C} f_{1}(z) z^{k} d z=\int_{C^{\prime}} f_{2}(z) z^{k} d z \quad(k=\cdots-2,-1,0,1,2, \cdots),
$$

then there exists a function $F(z)$ analytic in the annular region bounded by $C$ and $C^{\prime}$, continuous in the corresponding closed region, and which on $C$ and $C^{\prime}$ coincides with $f_{1}(z)$ and $f_{2}(z)$ respectively.

It is sufficient to establish Theorem XIV in the case that $C$ is a circle. For if the theorem is true in that case, we shall prove it to be true in the general 
case. Let $z=\psi(x), x=\phi(z)$ denote as usual the functions which map the interior of $C$ onto the interior of the unit circle $\gamma$ in the $x$-plane. Since $\phi(0)=0$, the curve $C^{\prime}$ corresponds to an analytic Jordan curve $\gamma^{\prime}$ in whose interior the origin $x=0$ lies.

Conditions (28) lead to the equations

$$
\begin{aligned}
\int_{C} f_{1}(z)[\phi(z)]^{k} \phi^{\prime}(z) d z=\int_{C^{\prime}} f_{2}(z)[\phi(z)]^{k} \phi^{\prime}(z) d z \\
\\
\quad(k=\cdots-2,-1,0,1,2, \cdots) .
\end{aligned}
$$

For the function $[\phi(z)]^{k} \phi^{\prime}(z)$ is analytic in the closed region bounded by $C$ and $C^{\prime}$, hence in that closed region can be uniformly expanded in a series of polynomials in $z$ and $1 / z{ }^{*}$ This expansion can be integrated term by term on $C$ or $C^{\prime}$, after multiplication through by $f_{1}(z)$ or $f_{2}(z)$. Computation of the two members of (29) by the use of this series makes their equality evident in the light of (28).

Equations (29) are precisely the equations

$$
\int_{\gamma} f_{1}[\psi(x)] x^{k} d x=\int_{\gamma^{\prime}} f_{2}[\psi(x)] x^{k} d x \quad(k=\cdots-2,-1,0,1,2, \cdots),
$$

sufficient for the existence of a function $f(x)$ analytic in the annular region bounded by $\gamma$ and $\gamma^{\prime}$, continuous in the corresponding closed region, and equal to $f_{1}[\psi(x)]$ and $f_{2}[\psi(x)]$ on $\gamma$ and $\gamma^{\prime}$; this is sufficient for the existence of the function $F(z)$ of the theorem.

It remains, then, to prove Theorem XIV when $C$ is a circle. Consider the formal Taylor development of the function $f_{1}(z)$ :

$$
f_{1}(z) \sim a_{0}+a_{1} z+a_{2} z^{2}+\cdots, \quad a_{k}=\frac{1}{2 \pi i} \int_{C} \frac{f_{1}(z)}{z^{k+1}} d z .
$$

This series converges interior to $C$, defining a function $F_{1}(z)$ analytic interior to $C$, and the series converges uniformly on any curve $\Gamma$ interior to $C$. Thus if $\Gamma$ is an arbitrary rectifiable Jordan curve interior to $C$ and includes in its interior the origin, we have

$$
a_{k-1}=\int_{C} f_{1}(z) z^{-k} d z=\int_{\Gamma} F_{1}(z) z^{-k} d z \quad(k=1,2,3, \cdots) .
$$

Hence the function $f_{z}(z)=F_{1}(z)$ is continuous on $C^{\prime}$ and satisfies the conditions

\footnotetext{
- This is very easy to prove by writing the function involved as the sum of two functions, given by Cauchy's integral taken over $C$ and $C^{\prime}$ respectively. The one function is analytic on and interior to $C$, the other on and exterior to $C^{\prime}$.
} 


$$
\begin{aligned}
& \int_{C^{\prime}}\left[f_{2}(z)-F_{1}(z)\right] z^{-k} d z=\int_{C^{\prime}} f_{2}(z) z^{-k} d z-\int_{C} f_{1}(z) z^{-k} d z=0 \\
&(k=1,2,3, \cdots) .
\end{aligned}
$$

By Theorem XIII there exists a function $F_{2}(z)$ which is analytic exterior to $C^{\prime}$, continuous in the corresponding closed region, and which on $C^{\prime}$ coincides with $f_{2}(z)-F_{1}(z)$. The function $F_{2}(z)-f_{1}(z)$ is continuous on $C$. From the relations

$$
\begin{aligned}
\int_{C} F_{2}(z) z^{k} d z & =\int_{C^{\prime}} F_{2}(z) z^{k} d z=\int_{C^{\prime}} f_{2}(z) z^{k} d z-\int_{C^{\prime}} F_{1}(z) z^{k} d z \\
& =\int_{C^{\prime}} f_{2}(z) z^{k} d z=\int_{C} f_{1}(z) z^{k} d z \quad(k=0,1,2, \cdots),
\end{aligned}
$$

we deduce by Theorem XII the existence of a function $\Phi(z)$ analytic interior to $C$, continuous in the corresponding closed region, and coinciding on $C$ with $F_{2}(z)-f_{1}(z)$ :

$$
\Phi(z)=F_{2}(z)-f_{1}(z), \quad z \text { on } C .
$$

We have, however,

$$
\begin{aligned}
-\int_{C} \Phi(z) z^{k} d z=\int_{C}\left[F_{2}(z)-\Phi(z)\right] z^{k} d z & =\int_{C} f_{1}(z) z^{k} d z \\
(k & =-1,-2,-3, \cdots),
\end{aligned}
$$

so that the two functions $-\Phi(z)$ and $F_{1}(z)$ have the same coefficients in their Taylor development about the origin and are therefore identical.

The function $F_{2}(z)-\Phi(z)$ is then analytic interior to the annular region bounded by $C$ and $C^{\prime}$, continuous in the corresponding closed region, by (30) equals $f_{1}(z)$ on $C$, and by the definition of $F_{2}(z)$ equals $f_{2}(z)$ on $C^{\prime}$.

Theorem XIV, whose proof is now complete, can be extended to regions of higher connectivity:

TheOREM XV. Let $R$ be the region bounded by an analytic Jordan curve $C_{0}$ and by non-intersecting analytic Jordan curves $C_{1}, C_{2}, \cdots, C_{n}$ lying interior to $C_{0}$. If the function $f(z)$ is continuous on $C$, the complete boundary of $R$, then a necessary and sufficient condition that there exist a function $F(z)$ analytic in $R$, continuous in the corresponding closed region, and equal to $f(z)$ on $C$, is 


$$
\begin{array}{lr}
\int_{C} f(z) z^{k} d z=0 & (k=0,1,2, \cdots), \\
\int_{C} f(z)\left(z-z_{i}\right)^{k} d z=0 & (i=1,2, \cdots, n ; k=1,2,3, \cdots),
\end{array}
$$

where $z_{i}$ is an arbitrary fixed point interior to $C_{i}$. The integrals in (31) are to be taken over the complete boundary of $R$, in the positive sense on that boundary.

The proof of Theorem XV is similar to the proof of Theorem XIV and is omitted. Theorem XV remains true if the Jordan curves $C_{i}$ are no longer required to be analytic, provided they are regular in the sense of Osgood,* and provided the function $f(z)$ satisfies a Lipschitz condition on $C$. The proof of this new theorem is likewise fairly simple, as an application of the theorem of Plemelj used in I, p. 167. It will be noticed too that in the proofs of the theorems given we need not require that the curves used be analytic; it is sufficient if the derivative $\phi^{\prime}(z)$ of the mapping function used in each case is continuous in the closed region which we map.

The existence of other theorems which lie not far away is obvious; we give a single example related to Theorem XIV:

TheOREM XVI. Let the analytic Jordan curve $C^{\prime}$ lie interior to the analytic Jordan curve $C$, let the crigin lie interior to $C^{\prime}$, and let the functions $f_{1}(z)$ and $f_{2}(z)$ be defined and continuous on $C$ and $C^{\prime}$ respectively. Then a necessary and sufficient condition for the existence of a function $F(z)$ analytic interior to $C$, continuous in the corresponding closed region, and coinciding on $C$ and $C^{\prime}$ with $f_{1}(z)$ and $f_{2}(z)$ respectively, is

$$
\begin{array}{lr}
\int_{C} f_{1}(z) z^{k} d z=\int_{C^{\prime}} f_{2}(z) z^{k} d z=0 & (k=0,1,2, \cdots), \\
\int_{C} f_{1}(z) z^{k} d z=\int_{C^{\prime}} f_{2}(z) z^{k} d z & (k=-1,-2,-3, \cdots) .
\end{array}
$$

Theorems XII-XVI have obvious application to expansions in terms of polynomials, particularly in connection with such theorems as IX, which do not demand analyticity for the development of a given function.

\footnotetext{
* Funktionentheorie, Leipzig, 1912, p. 51.

Harvard UniverstTy,

Caubridge, Mass.
} 